Pacific Journal of Mathematics

A HOMOGENEOUS EBERLEIN COMPACT SPACE WHICH IS 


\title{
A HOMOGENEOUS EBERLEIN COMPACT SPACE WHICH IS NOT METRIZABLE
}

\author{
JAN VAN MILL
}

We give an example of a first countable, hereditarily normal, homogeneous Eberlein compact space which is not metrizable. This answers a question of A. V. Arhangel'skiv.

1. Introduction. A compact Hausdorff space is called Eberlein compact, if it is homeomorphic to a weakly compact subset of a Banach space. For information concerning Eberlein compact spaces, see [1], [3], [5] and [7].

If $X$ is Eberlein compact, then $X$ is metrizable if $X$ satisfies the countable chain condition, [2], [5], or if $X$ is linearly orderable, [4]. In view of these facts, the following question due to Arhangel'skii [3, p. 91 problem 5], is quite natural: is there a nonmetrizable homogeneous ${ }^{1}$ Eberlein compact space? The aim of this paper is to construct such an example which in addition is zerodimensional, first countable and hereditarily normal. The symbol " $X \approx Y$ " means that $X$ and $Y$ are homeomorphic spaces. I am indebted to Mary Ellen Rudin for spotting some inaccuracies in an earlier version of this paper.

2. Preliminaries. A family $\mathscr{F}$ of subsets of a topological space $X$ is called separating provided that for any distinct $x, y \in X$ there is an $F \in \mathscr{F}$ such that either $x \in F$ and $y \notin F$ or $y \in F$ and $x \notin F$. The family $\mathscr{F}$ is called point-finite if each $x \in X$ belongs to at most finitely many elements of $\mathscr{F}$. It is called $\sigma$-point-finite if $\mathscr{F}=\bigcup_{n=1}^{\infty} \mathscr{F}_{n}$, where each $\mathscr{F}_{n}$ is point-finite.

The following purely topological characterization of Eberlein compacta, due to Rosenthal [8], is convenient for topologists.

THEOREM 2.1. A compact Hausdorff space is Eberlein compact iff it has a $\sigma$-point-finite separating family of open $F_{\sigma}$-subsets.

Let $C$ denote the usual Cantor set in $[0,1]$ (notice that $0 \in C$ ) and let $X$ be any space. Topologize $X \times C$ in the following way:

(a) a basic neighborhood of a point $\langle x, 0\rangle$ has the form

$$
(U \times C)-(\{x\} \times D),
$$

where $U \subset X$ is open, contains $x$ and $D \subset C-\{0\}$ is compact;

${ }^{1}$ A space $X$ is called homogeneous provided that for any two points $x, y \in X$ there is an autohomeomorphism $h: X \rightarrow X$ with $h(x)=y$. 
(b) a basic neighborhood of a point $\langle x, c\rangle$ where $c>0$ has the form

$$
\{x\} \times U,
$$

where $U \subset C-\{0\}$ is an open neighborhood of $c$.

The topological space we obtain in this way will be denoted by $X(C)$. Observe that the projection $\pi: X(C) \rightarrow X$ onto the first coordinate is continuous. In addition, the function $f: X \rightarrow X(C)$ defined by $f(x)=\langle x, 0\rangle$ is an embedding.

LEMMA 2.2. (1) $X(C)$ is compact Hausdorff iff $X$ is compact Hausdorff,

(2) $X(C)$ is first countable iff $X$ is first countable,

(3) $X(C)$ is Eberlein compact iff $X$ is Eberlein compact.

Proof. (1) We only need to show that $X(C)$ is compact if $X$ is. Let $\mathscr{U}$ be an open cover of $X(C)$ by basis elements. Finitely many elements of $\mathscr{C}$ cover $X \times\{0\}$ and the remaining part of $X(C)$ consists of finitely many compact sets. We conclude that $\mathscr{C}$ has a finite subcover.

Observe that (2) is trivial and that for (3) we only need to show that $X(C)$ is Eberlein compact if $X$ is Eberlein compact (closed subsets of Eberlein compacta are Eberlein compact). To this end, let $\mathscr{F}=\bigcup_{n=1}^{\infty} \mathscr{F}_{n}$ be a separating family of open $\mathscr{F}_{0}$-subsets of $X$ such that for all $n$ the family $\mathscr{F}_{n}$ is point-finite. In addition, let $\left\{C_{m}: m=1,2, \cdots\right\}$ be a countable basis for $C-\{0\}$ consisting of compact open sets. For all $n, m \in N$ define

$$
\tilde{\mathscr{F}}_{n}=\left\{F \times C: F \in \mathscr{F}_{n}\right\},
$$

and

$$
\mathscr{C}_{m}=\left\{\{x\} \times C_{m}: x \in X\right\}
$$

respectively. Observe that both $\tilde{\mathscr{F}}_{n}$ and $\mathscr{C}_{m}$ are point-finite, that $\widetilde{F}_{n}$ consists of open $F_{o}$-subsets of $X(C)$ and that $\mathscr{C}_{m}$ consists of clopen ( = closed and open) subsets of $X(C)$. Since trivially,

$$
\mathscr{G}=\bigcup_{n=1}^{\infty} \tilde{\mathscr{F}}_{n} \cup \bigcup_{m=1}^{\infty} \mathscr{C}_{m}
$$

is separating, Theorem 2.1 implies that $X(C)$ is Eberlein compact.

3. The example. Let $X$ be any space. Define $X_{1}=X$ and $X_{n+1}=X_{n}(C)$. The projection from $X_{n+1}$ onto its first coordinate is 
denoted by $f_{n, x}$. Put

$$
\tilde{X}=\lim _{\leftarrow}\left(X_{n}, f_{n, X}\right)
$$

(i.e., $\tilde{X}=\left\{x \in \prod_{n=1}^{\infty} X_{n}: f_{n, X}\left(x_{n+1}\right)=x_{n}\right.$ for all $\left.n \in N\right\}$ ). Let $\pi_{n, X}: \tilde{X} \rightarrow X_{n}$ be the projection. Observe that

$$
\pi_{n, X}=f_{n, X} \circ \pi_{n+1, X} .
$$

Lemma 3.1. (1) If $A \subset X$, then $\tilde{A} \approx \pi_{1, X}^{-1}(A) \subset \tilde{X}$.

(2) $\tilde{X}_{n} \approx \widetilde{X}$ for all $n \in N$.

Proof. Obvious.

We claim that $\widetilde{C}$ is homogeneous, nonmetrizable and Eberlein compact. By a repeated application of Lemma 2.2(3) it follows that each $C_{n}$ is Eberlein compact. Consequently, by Theorem 2.1,

$$
\prod_{n=1}^{\infty} C_{n}
$$

is Eberlein compact which implies that $\widetilde{C}$ is Eberlein compact, being a closed subspace of $\prod_{n=1}^{\infty} C_{n}$. Similarly, each $C_{n}$ is first countable and consequently, $\widetilde{C}$ is first countable. It is clear that $\widetilde{C}$ is not metrizable, since it maps onto the nonmetrizable space $C_{2}\left(C_{2}\right.$ is not metrizable since it contains an uncountable family of pairwise disjoint nonempty open subsets). Obviously, $\widetilde{C}$ is zerodimensional.

TheOREM 3.2. $\widetilde{C}$ has the property that all of its nonempty clopen subspaces are homeomorphic (hence $\widetilde{C}$ is strongly homogeneous in the sense of [8]).

Proof. By induction on $n$ we will show that $\pi_{n, C}^{-1}(U) \approx \widetilde{C}$ for all nonempty clopen $U \subset C_{n}$. This is clearly true for $n=1$ since all nonempty clopen subsets of $C$ are homeomorphic to $C$ which implies that

$$
\pi_{1, C}^{-1}(U) \approx \tilde{U} \approx \widetilde{C}
$$

for all clopen $U \subset C_{1}$ (Lemma 3.1(1)). Now suppose the statement to be true for $n$ and take a nonempty clopen $U \subset C_{n+1}$ arbitrarily. If $U \cap\left(C_{n} \times\{0\}\right)=\varnothing$ then $U$ is homeomorphic to $C$ by definition of the topology of $C_{n+1}$. Consequently, by Lemma 3.1(1), (2) it then follows that

$$
\pi_{n+1, c}^{-1}(U) \approx \widetilde{U} \approx \widetilde{C} .
$$

Therefore assume that $U \cap\left(C_{n} \times\{0\}\right) \neq \varnothing$. By definition of the 
topology of $C_{n+1}$ there is a finite $F \subset C_{n}$ and for each $x \in F$ a clopen $S_{x} \subset C$ not containing 0 such that $F \times\{0\} \subset V=U \cap\left(C_{n} \times\{0\}\right)$ while moreover

$$
E=(V \times C)-\left(\bigcup_{x \in F}\{x\} \times S_{x}\right) \subset U .
$$

For each $x \in F$ let $h_{x}: C-S_{x} \rightarrow C$ be a homeomorphism such that in a fixed neighborhood of 0 each $h_{x}$ is the identity. Define $h: E \rightarrow V \times$ $C$ by

$$
\left\{\begin{array}{lll}
h(\langle a, b\rangle)=\langle a, b\rangle & \text { if } & a \notin F \\
h(\langle a, b\rangle)=\left\langle a, h_{a}(b)\right\rangle & \text { if } & a \in F
\end{array}\right.
$$

Clearly, $h$ is a homeomorphism. Therefore

$$
\pi_{n+1, C}^{-1}(E) \approx \pi_{n}^{-1}(V) \approx \widetilde{C},
$$

by induction hypothesis. Put $G=U-E$. Then $G$ is a clopen subset of $C_{n+1}$ which misses $C_{n} \times\{0\}$. If $G=\varnothing$ then we are done, and if $G \neq \varnothing$ then observe that

$$
\pi_{n+1, c}^{-1}(G) \approx \widetilde{C}
$$

since $G \approx C$ (cf. the above remarks). Consequently, $\pi_{n+1, C}^{-1}(U)$ is the disjoint union of two clopen copies of $\widetilde{C}$, hence is itself homeomorphic to $\widetilde{C}$ since $C$ is the disjoint union of two clopen copies of itself. This completes the induction.

Now let $A \subset \widetilde{C}$ be clopen and nonempty. There is clearly an index $n \in N$ and a nonempty clopen $B \subset C_{n}$ such that

$$
\pi_{n, C}^{-1}(B)=A \text {. }
$$

Therefore $A=\pi_{n, C}^{-1}(B) \approx \widetilde{C}$.

The above theorem shows that $\widetilde{C}$ is homogeneous, for any zerodimensional strongly homogeneous first countable space $X$ is homogeneous. This is well-known and for completeness sake we will include the trivial proof. Take $x, y \in X$. Since $X$ is first countable, there is a clopen neighborhood basis $\left\{V_{n}: n \in N\right\}$ for $x$ and a clopen neighborhood basis $\left\{W_{n}: n \in N\right\}$ for $y$ such that

(1) $V_{1}=W_{1}=X$,

(2) $V_{n+1}$ is properly contained in $V_{n}$, and

(3) $W_{n+1}$ is properly contained in $W_{n}$.

For each $n \in N$ let $h_{n}: V_{n}-V_{n+1} \rightarrow W_{n}-W_{n+1}$ be any homeomorphism. The function $h: X \rightarrow X$ defined by

$$
\left\{\begin{array}{l}
h(x)=y \\
h(a)=h_{n}(a) \quad \text { if } \quad a \in V_{n}-V_{n+1}
\end{array}\right.
$$


is clearly a homeomorphism mapping $x$ onto $y$.

REMARK 3.3. It is not by accident that our example is first countable. By [5,4.3] every Eberlein compact space is first countable at a dense set of points, consequently, a homogeneous Eberlein compact space must be first countable. Notice however that we used the first countability of $\widetilde{C}$ to show it is homogeneous.

4. $\widetilde{C}$ is hereditarily normal. In this section we will show that $\widetilde{C}$ is a continuous image of a compact linearly orderable topological space. This implies that $\widetilde{C}$ is hereditarily normal (even monotonically normal).

Let $L_{1}=C$ and let $L_{2}=C \times C$ with topology generated by the lexicographical ordering. Let $g_{1}: L_{2} \rightarrow L_{1}$ be the projection onto the first coordinate. Observe that $g_{1}$ is order preserving. Let $\psi_{1}: L_{1} \rightarrow$ $C_{1}$ be the identity and let $h: C \rightarrow C$ be an arbitrary onto map such that

$$
h(0)=0 \text { and } h(1)=0 .
$$

Define $\psi_{2}: L_{2} \rightarrow C_{2}$ by

$$
\psi_{2}(\langle a, b\rangle)=\langle a, h(b)\rangle .
$$

Because $h(0)=0=h(1), \psi_{2}$ is continuous.

It is easily seen that the diagram

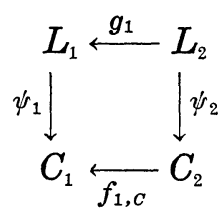

commutes. Suppose that we have defined $L_{n}$ and $\psi_{n}$. Let $L_{n+1}=$ $L_{n} \times C$ with topology generated by the lexicographical ordering and let $g_{n}: L_{n+1} \rightarrow L_{n}$ be the projection. Define $\psi_{n+1}: L_{n+1} \rightarrow C_{n+1}$ by $\psi_{n+1}(\langle a, b\rangle)=\langle a, h(b)\rangle$, where $h$ is defined as above. Observe that $g_{n}$ is order preserving and that

$$
f_{n, C} \circ \psi_{n+1}=\psi_{n} \circ g_{n}
$$

Put $L=\lim _{\leftarrow}\left(L_{n}, g_{n}\right)$. Since the maps $g_{n}$ are all order preserving, $L$ can be $\overleftarrow{\leftarrow}$ ordered in a natural way (It is easy to describe the ordering of $L$. Alternatively, the orderability theorems given in [6] or [9] are also easily applied.). By (1), the space $L$ maps onto $\widetilde{C}$ so that $\widetilde{C}$ is hereditarily normal.

As was pointed out to me by Dave Lutzer, it is also easily seen 


\section{that $\widetilde{C}$ is hereditarily paracompact, since $L$ is a first countable compact LOTS and $L$ maps onto $\widetilde{C}$.}

\section{REFERENCES}

1. D. Amir and J. Lindenstrauss, The structure of weakly compact sets in Banach spaces, Ann. of Math., 88 (1968), 34-46.

2. A. V. Arhangel'skir, On some topological spaces that occur in functional analysis, Russian Math. Surveys, 31 (1976), 14-30.

3. - Structure and classification of topological spaces and cardinal invariants, Russian Math. Surveys, 33 (1978), 33-96.

4. H. R. Bennett, D. J. Lutzer and J. M. van Wouwe, Linearly ordered Eberlein compact spaces, Top. Appl., 12 (1981), 11-18.

5. Y. Benyamini, M. E. Rudin and M. Wage, Continuous images of weakly compact subsets of Banach spaces, Pacific J. Math., 70 (1977), 309-324.

6. J. de Groot and P. S. Schnare, A topological characterization of products of compact totally ordered spaces, General Topology and Appl., 2 (1972), 67-73.

7. J. Lindenstrauss, Weakly compact sets, their topological properties and the Banach spaces they generate, Annals of Math. Studies 69, Princeton University Press, (1972), 235-273.

8. J. van Mill, Characterization of some zero-dimensional separable metric spaces, Trans. Amer. Math. Soc., 264 (1981), 205-215.

9. J. van Mill and E. Wattel, Selections and orderability, to appear in Proc. Amer. Math. Soc., 83 (1981), 601-605.

10. H. P. Rosenthal, The hereditary problem for weakly compactly generated Banach spaces, Comp. Math., 28 (1974), 83-111.

Received February 11, 1981 and in revised form July 6, 1981.

Subfaculteit Wiskunde

VRije Universiteit

De Boelelaan 1081

Amsterdam, The Netherlands 


\section{PACIFIC JOURNAL OF MATHEMATICS}

\section{EDITORS}

DONALD BABBITT (Managing Editor)

J. DUGUNDJI

University of California

Los Angeles, California 90024

Hugo Rossi

University of Utah

Salt Lake City, UT 84112

C. C. Moore and Arthur Agus

Department of Mathematics

University of Southern California

Los Angeles, California 90007

R. FinN and J. Milgram

Stanford University

Stanford, California 94305

University of California

Berkeley, CA 94720

\section{ASSOCIATE EDITORS}
R. ARNES
E. F. BeCKenBaCH
B. H. NEUMANN
F. WoLF
K. YoshidA

\section{SUPPORTING INSTITUTIONS}

UNIVERSITY OF ARIZONA

UNIVERSITY OF BRITISH COLUMBIA

CALIFORNIA INSTITUTE OF TECHNOLOGY

UNIVERSITY OF CALIFORNIA

MONTANA STATE UNIVERSITY

UNIVERSITY OF NEVADA, RENO

NEW MEXICO STATE UNIVERSITY

OREGON STATE UNIVERSITY
UNIVERSITY OF OREGON

UNIVERSITY OF SOUTHERN CALIFORNIA

STANFORD UNIVERSITY

UNIVERSITY OF HAWAII

UNIVERSITY OF TOKYO

UNIVERSITY OF UTAH

WASHINGTON STATE UNIVERSITY

UNIVERSITY OF WASHINGTON 


\section{Pacific Journal of Mathematics}

\section{Vol. 101, No. $1 \quad$ November, 1982}

Natália Bebiano, On the evaluation of permanents $\ldots \ldots \ldots \ldots \ldots \ldots \ldots \ldots$

David Borwein and Bruce Brigham Watson, Tauberian theorems between

the logarithmic and Abel-type summability methods $\ldots \ldots \ldots \ldots \ldots \ldots 11$

Leo George Chouinard, II, Hermite semigroup rings $\ldots \ldots \ldots \ldots \ldots \ldots \ldots$

Kun-Jen Chung, Remarks on nonlinear contractions $\ldots \ldots \ldots \ldots \ldots \ldots . \ldots 4$

Lawrence Jay Corwin, Representations of division algebras over local

fields. II ......................................... 49

Mahlon M. Day, Left thick to left lumpy—a guided tour $\ldots \ldots \ldots \ldots \ldots 71$

M. Edelstein and Mo Tak Kiang, On ultimately nonexpansive

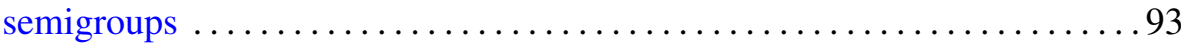

Mary Rodriguez Embry, Semigroups of quasinormal operators . ........ 103

William Goldman and Morris William Hirsch, Polynomial forms on

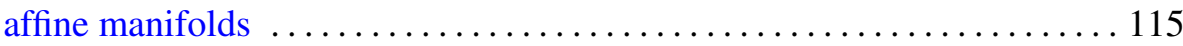

S. Janakiraman and T. Soundararajan, Totally bounded group topologies

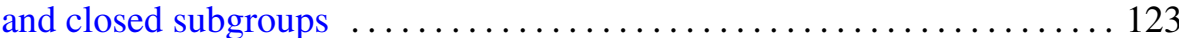

John Rowlay Martin, Lex Gerard Oversteegen and Edward D.

Tymchatyn, Fixed point set of products and cones $\ldots \ldots \ldots \ldots \ldots \ldots 133$

Jan van Mill, A homogeneous Eberlein compact space which is not metrizable ........................................ 141

Steven Paul Plotnick, Embedding homology 3-spheres in $S^{5} \ldots \ldots \ldots \ldots 147$

Norbert Riedel, Classification of the $C^{*}$-algebras associated with minimal rotations

Benedict Seifert, Combinatorial and geometric properties of weight systems of irreducible finite-dimensional representations of simple split Lie algebras over fields of 0 characteristic

James E. Simpson, Dilations on locally convex spaces

Paolo M. Soardi, Schauder bases and fixed points of nonexpansive mappings

Yoshio Tanaka, Point-countable $k$-systems and products of $k$-spaces

Fausto A. Toranzos, The points of local nonconvexity of starshaped sets . . . 209

Lorenzo Traldi, The determinantal ideals of link modules. I . . . . . . . . 215

P. C. Trombi, Invariant harmonic analysis on split rank one groups with applications

Shinji Yamashita, Nonnormal Blaschke quotients 